The Astrophysical Journal, 161:519-531, August 1970

(C) 1970 The University of Chicago. All rights reserved Printed in U S A.

\title{
THE FAINT END OF THE MAIN SEQUENCE
}

\author{
Jesse L. Greenstein and G. Neugebauer \\ Hale Observatories \\ California Institute of Technology, Carnegie Institution of Washington \\ AND \\ E. E. BECKLIN* \\ California Institute of Technology \\ Received 1970 January 19; revised 1970 February 16
}

\section{ABSTRACT}

New infrared observations of the two faintest known, late $M$ dwarfs, Wolf 359 and $+4^{\circ} 4048 \mathrm{~B}$ $(=\mathrm{VB} 10)$ provide accurate luminosities and moderately well-determined temperatures $\left(2500^{\circ}\right.$ and $2250^{\circ} \mathrm{K}$, respectively). The photometric observations are fitted to a blackbody energy distribution on the assumption that line and band blocking affect most of the spectrum below $1 \mu$; the temperature structure is taken as that of a gray body. The resulting data, together with Johnson's observations for dM4 and dM5 stars, which have been reanalyzed, calibrate the faint end of the main sequence, with results given in a table and a figure. The bolometric corrections are very large and increase steeply to $6 \mathrm{mag}$, so that the faintest known stars are, in fact, not very faint; Wolf 359 has $L=13 \times 10^{-4} L_{\odot}$, and VB 10 has $L=5 \times 10^{-4} L_{\odot}$. A statistical discussion of Luyten's faint red stars of large proper motion gives $L=4 \times 10^{-4} L_{\odot}$. With a conventional mass-luminosity relation, $\mathfrak{M} \geq 0.09 \mathfrak{M}_{\odot}$, for stars of known luminosity. Stars of still lower mass, such as L726-8, are difficult to interpret.

\section{INTRODUCTIGN: NEW DATA}

The faint end of the main sequence has remained largely unexplored because of the apparent and intrinsic faintness of even the nearest members of this class. The infrared brightness of the nearby flare star Wolf 359 (=Giclas 45-20), whose parallax is $p=$ 0 ".402, results in the surprisingly large color index $V-L=7.8$ mag. Such a color is exceeded only by that of long-period variables or of infrared stars. The low temperatures deduced near the faint end of the main sequence result in a large bolometric correction. $H, K, L$ magnitudes, on the Johnson system, of Wolf 359 were measured in 1968 April and May with the Palomar 200-inch reflector. In addition, the faintest known star, $+4^{\circ} 4048 \mathrm{~B}=$ VB 10 (Van Biesbroeck 1944, 1961), $p=0$ ".168, was observed in 1968 September (100-inch) and November (200-inch). VB 10 has $V-L=9.6 \mathrm{mag}$, also extremely red. Wolf 359 was observed in a spectroscopic flare by Greenstein and Arp (1969), and VB 10 by Herbig (1956). Near-infrared colors of VB 10 were measured by Kron (1958). Johnson's (1965a) colors of Wolf 359 are also included in the total data assembled in Table 1; the conversion of magnitude to flux is close to that of Johnson $(1965 b)$ with minor recalibration by Becklin (1968) and Neugebauer. No evidence of variability of these two dMe stars in the (1.6-3.5)- $\mu$ region has been found. The measures, plotted in Figure 1, define a large part of the total flux of these stars and determine the bolometric luminosity accurately. They provide reasonably good limits on the effective temperature and radii.

The large $V-L$ colors, however, may produce an illusion of low temperature and exaggerate the possible accuracy of temperature determination. Although full details of the spectra are not known over this long wavelength span, it is certain that $\mathrm{TiO}$ bands absorb heavily in the $V$ - and $R$-bands, and appreciably in the $I$-band. Theoretical atmospheric models show strong $\mathrm{H}_{2} \mathrm{O}$ and $\mathrm{CO}$ absorption, with a window at $1.6 \mu$,

\footnotetext{
* Present address: Smithsonian Astrophysical Observatory, Cambridge, Mass.
} 
where a relatively strong peak exists in $\mathrm{M}$ giants. A grating spectrum of W359 by Hyland (private communication) shows weak $\mathrm{CO}$ bands in the 1.6- and 2.2- $\mu$ regions. Wing and Ford (1969) find strong TiO and an unidentified band near $1 \mu$. Titanium oxide is known to be very strong in the M2-M6 dwarfs, in the blue and red. Furthermore, the strong low-excitation metal lines must produce large depressions in the $U B V$ regions. Until detailed model atmospheres, including atomic lines and molecular bands, are compared

TABLE 1

OBSERVED MAGNITUDES AND FLUXES

\begin{tabular}{|c|c|c|c|c|c|c|c|c|}
\hline \multirow[b]{2}{*}{ Band } & \multirow{2}{*}{$\begin{array}{c}\lambda \\
(\mu)\end{array}$} & \multirow{2}{*}{$\begin{array}{c}\lambda^{-1} \\
\left(\mu^{-1}\right)\end{array}$} & \multicolumn{2}{|c|}{$m_{\nu}$} & \multicolumn{2}{|c|}{$M_{*}$} & \multicolumn{2}{|c|}{$\left(\mathrm{W} \mathrm{m}^{-2} \mathrm{~Hz}^{-1}\right)$} \\
\hline & & & W359 & VB 10 & W359 & VB 10 & W359 & VB 10 \\
\hline 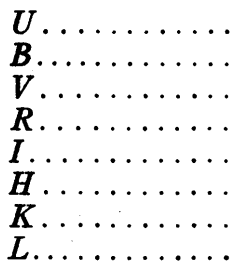 & $\begin{array}{l}0.36 \\
0.44 \\
0.55 \\
0.70 \\
0.90 \\
1.65 \\
2.2 \\
3.4\end{array}$ & $\begin{array}{l}2.78 \\
2.27 \\
1.82 \\
1.43 \\
1.11 \\
0.61 \\
0.45 \\
0.28\end{array}$ & $\begin{array}{l}16.8 \\
15.6 \\
13.6 \\
10.9(J) \\
8.4(J) \\
6.5 \\
6.1 \\
5.8\end{array}$ & $\begin{array}{l}20.1(K) \\
17.8(K) \\
\ldots .2 \\
12.4(K) \\
9.4 \\
8.75 \\
8.24\end{array}$ & $\begin{array}{r}19.8 \\
18.6 \\
16.6 \\
13.9 \\
11.4 \\
9.5 \\
9.1 \\
8.8\end{array}$ & $\begin{array}{r}21.2 \\
18.9 \\
13.5 \\
10.5 \\
9.9 \\
9.3\end{array}$ & $\begin{array}{l}-29.45 \\
-28.59 \\
-27.86 \\
-26.87 \\
-25.97 \\
-25.59 \\
-25.64 \\
-25.83\end{array}$ & $\begin{array}{l}-30.39 \\
-29.53 \\
-27.61 \\
-26.75 \\
-26.68 \\
-26.87\end{array}$ \\
\hline
\end{tabular}

Note. $-J=$ Johnson; $K=$ Kron.

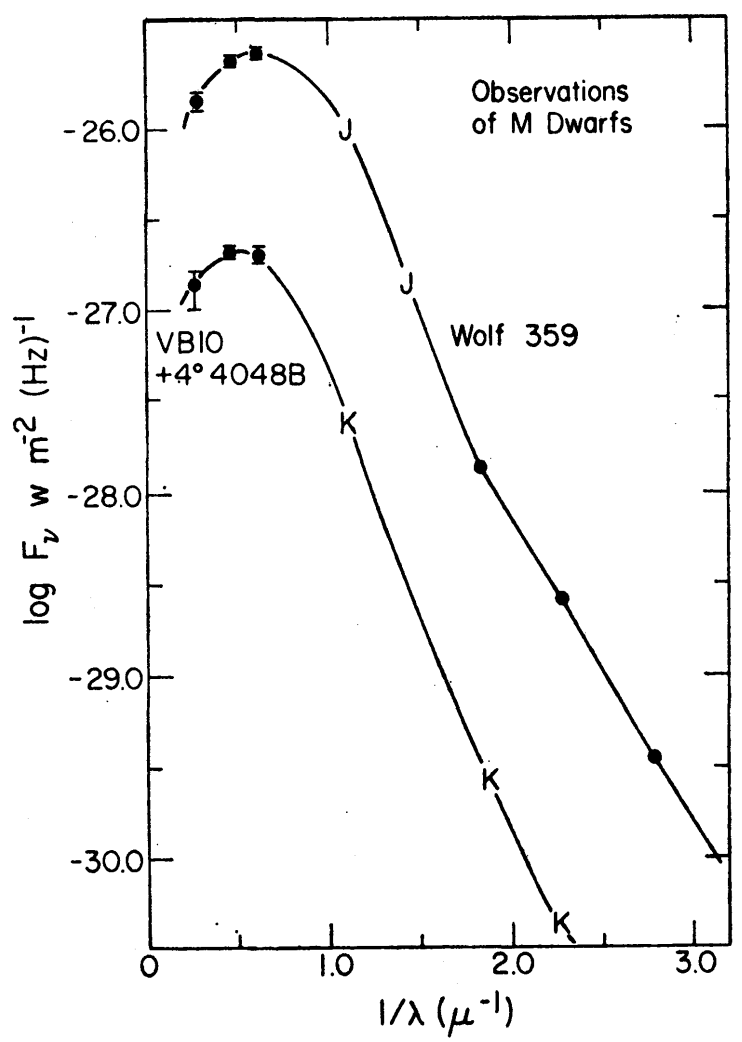

Fig. 1.-The observed fluxes, $\log _{10} F_{\nu}$, in broad-band filters for Wolf 359 ( $J$, measures by Johnson), and VB 10 ( $K$, measures by Kron). Error bars at JKL are based on internal uncertainties. Frequency scale is inverse microns. Curves drawn have been copied on Figs. 2 and 3 , for curve-fitting. 
with spectra, it is, in fact, very difficult to provide an accurate temperature scale. The presently available models do not include atomic lines, and do not agree well with observation. Consequently, the available data have been treated in what seems to be a conservative manner.

\section{AN ELEMENTARY THEORY OF THE DEPRESSION OF THE CONTINUUM AND THE DETERMINATION OF $T, L, R$}

If lines and bands severely depress large regions of the spectrum, the lower limit to the flux in a filter passband is the thermal emission at the boundary temperature, $T_{0}$. The bands and lines may not be conspicuous on spectra if located in a region of high band opacity or general opacity. We will attempt to fit blackbody curves of effective temperature $T_{e}$ to the observed energy distributions of $M$ dwarfs. The maximum allowable depression, $\phi_{\nu}$, can be computed if $T_{0}$ is known. The flux emitted cannot be less than

$$
\mathfrak{F}_{\nu}=\phi_{\nu}{ }^{-1} B_{\nu}\left(T_{e}\right)
$$

in regions of high opacity. Assume a gray-body model in radiative equilibrium, for which $T_{0}=T_{e} / 1.233$. (In fact, $T_{0}$ may be much smaller, according to present models, but the two errors compensate, since it is unlikely that a passband is completely covered by saturated bands and lines.) To keep the integrated actual flux the same as a blackbody of the given effective temperature, we may assume that the energy at wavelengths of high opacity is emitted at a radius $R_{0}$ different from that of the star, $R$. As an ad hoc assumption, we assume that

$$
R^{2} \sigma T_{e}^{4}=R_{0}^{2} \sigma T_{0}^{4}
$$

Then the depression at any frequency becomes

$$
\phi_{\nu}=\frac{R^{2} B_{\nu}\left(T_{e}\right)}{R_{0}^{2} B_{\nu}\left(T_{0}\right)}
$$

which reduces, for the gray-body case, to

$$
\phi_{\nu}=\left(\frac{1}{1.233}\right)^{4} \frac{\exp \left(h \nu / k T_{0}\right)-1}{\exp \left(h \nu / k T_{e}\right)-1} .
$$

The tabulated values of $\log \phi_{\nu}$ in Table 2 give the behavior to be expected in curve fitting. The negative values of $\log \phi_{\nu}$ arise from the requirement of correct total flux. Implausibly high $T_{e}$ will be recognized if the observed value of $\log B_{\nu}\left(T_{e}\right) / F_{\nu}$ exceeds $\log \phi_{\nu}$, a situation which usually occurs at short wavelengths. The allowed depression is very large; in the $B$-filter band, compared to the $K$-filter band, at $2500^{\circ} \mathrm{K}$, the change in $\log \phi_{\nu}$ is 1.0 ; i.e., a blocking of $2.5 \mathrm{mag}$ may occur. In the $I$-filter, as compared with $K$, the change of $\log \phi_{\nu}$ is 0.28 , and a blocking of only $0.5 \mathrm{mag}$ is permissible. Of course, if the real stellar model has a lower $T_{0} / T_{e}$, the effects may be even larger.

The flux $F_{\nu}$ at the Earth is determined from the apparent magnitudes as $F_{\nu}=C_{\nu}$ $10^{-0.4 m_{\nu}}$, where $C_{\nu}$ is the calibration constant of the photometric system. For a blackbody of radius $R$ and distance $r$, we have

$$
F_{\nu}=\frac{4 \pi R^{2} \pi B_{\nu}\left(T_{e}\right)}{4 \pi r^{2}}
$$

When $T_{e}$ is determined, the radius and luminosity are given. If $r$ is in parsecs, the radius in solar units is

$$
\log R / R_{\odot}=+7.40-\frac{1}{2} \log B_{v}\left(T_{e}\right) / F_{\nu}+\log r_{\mathrm{pc}} .
$$


For individual stars with $F_{\nu}$ observed, equation (5b) is used. We could also use mean apparent or absolute magnitudes for groups of stars by simple transformations of equation (5b) and computations of $\pi F_{\nu}$. In practice, the vertical shift, $\log B_{\nu}\left(T_{e}\right) / F_{\nu}$ is determined by fitting the mean observed $\log F_{\nu}$ curve to a set of blackbody curves (computed in steps of $125^{\circ} \mathrm{K}$ ) while keeping the $\log \phi_{\nu}$ in mind. The vertical shift is read in such a way as to keep the same area under the $B_{\nu}(T)$ and $F_{\nu}$ curves.

The unknown depressions by $\mathrm{TiO}$ and other atoms, and the high transparency of $\mathrm{H}_{2} \mathrm{O}$ and $\mathrm{CO}$ near the energy maximum make available models nearly useless in narrowing the range of $T$. Model atmospheres by Tsuji (quoted in Greenstein 1969) include TiO as well as $\mathrm{H}_{2} \mathrm{O}$ and $\mathrm{CO}$, but not convection effects. Tsuji finds high transparency at $\lambda<1.2 \mu$, which we do not believe justified by observations. Kandel (1969) notes the poor fit of theoretical and observed profiles of $\lambda 4227$ in K7 to M3 stars, where the line is far too weak and shallow. He invokes unknown sources of opacity (probably lines and bands). The effect is very large in the $U B V$ colors. Tsuji's model of a $4000^{\circ} \mathrm{K}$ star (near K7 V) predicts $B-V=+0.8, U-B=0.1$ (no atomic lines). The observed $\mathrm{K} 7$ colors are $+1.3,+1.1$, a differential blocking of +0.5 mag between $B$ and $V$, and +1.0 mag between $U$ and $B$. But from extrapolation of Table 2, we see that the expected $\phi_{\nu}$

TABLE 2

MAXIMUM FluX Deficiency, GRAY-BODy MODEL, $\log _{10} \phi_{\nu}$

\begin{tabular}{|c|c|c|c|c|c|c|c|c|}
\hline \multirow{2}{*}{$\begin{array}{c}1 / \lambda \\
\left(\mu^{-1}\right)\end{array}$} & \multicolumn{8}{|c|}{ EFfective Temperature $\left(10^{3}{ }^{\circ} \mathrm{K}\right)$} \\
\hline & 3.75 & 3.50 & 3.25 & 3.0 & 2.75 & 2.5 & 2.25 & 2.0 \\
\hline $\begin{array}{l}0.5 \ldots \ldots \\
1.0 \ldots \ldots \\
1.5 \ldots \ldots \\
2.0 \ldots \ldots \\
2.5 \ldots \ldots \\
3.0 \ldots \ldots\end{array}$ & $\begin{array}{l}-0.14 \\
+0.03 \\
+0.22 \\
+0.41 \\
+0.61 \\
+0.80\end{array}$ & $\begin{array}{l}-0.13 \\
+0.06 \\
+0.26 \\
+0.47 \\
+0.68 \\
+0.88\end{array}$ & $\begin{array}{l}-0.12 \\
+0.09 \\
+0.31 \\
+0.53 \\
+0.76 \\
+0.98\end{array}$ & $\begin{array}{l}-0.10 \\
+0.12 \\
+0.36 \\
+0.61 \\
+0.85 \\
+1.09\end{array}$ & $\begin{array}{l}-0.08 \\
+0.17 \\
+0.43 \\
+0.70 \\
+0.96 \\
+1.22\end{array}$ & $\begin{array}{l}-0.06 \\
+0.22 \\
+0.51 \\
+0.80 \\
+1.09 \\
+1.38\end{array}$ & $\begin{array}{l}-0.03 \\
+0.28 \\
+0.61 \\
+0.93 \\
+1.25 \\
+1.58\end{array}$ & $\begin{array}{l}+0.01 \\
+0.36 \\
+0.73 \\
+1.09 \\
+1.46 \\
+1.82\end{array}$ \\
\hline
\end{tabular}

corresponds only to +0.4 mag $(B$ to $V)$ and +0.5 mag ( $U$ to $B$ ) for a completely linecovered spectrum of a star with a gray-body temperature contrast. Thus even the coarsest colorimetric work requires $T_{e} / T_{0}>1.233$; Tsuji (quoted in Greenstein 1969) and Auman (1969), who includes convection, predict this.

We believe that the entire region below $1 \mu$ is heavily absorbed in M dwarfs. To obtain $T_{e}$ and its permissible range, we proceed in a "conservative" fashion. We give five values of $T_{e} ; T_{u}$ is the highest possible, and is limited by the unacceptably high values of $\phi$ required; $T_{1}$ is a plausible upper value, $T$ the best, $T_{2}$ a plausible lower value, and $T_{l}$ the lowest possible. At $T_{l}$ the $F_{\nu}$ fits on the $B_{\nu}\left(T_{e}\right)$ curve at $\lambda<1 \mu$ (i.e., there is no line or band blocking); and the observed maximum is displaced toward shorter wavelengths. Both $T_{l}$ and $T_{u}$ are easily recognizable, but the differences between $T_{1}, T$, and $T_{2}$ are matters of judgment. The temperature used in theoretical discussions is nearly always the midpoint, T. Figures 2 and 3 show the observed curves for W359 and VB 10 and the fitted $B_{\nu}(T)$, at the best vertical shift. Table 3 gives the resulting parameters of the stars. The maximum range is $\pm 250^{\circ}$, the probable range $\pm 125^{\circ}$. To compute ${ }^{1}$ the bolometric magnitudes, $M_{\text {bol }}$, we have used $\left(M_{\mathrm{bol}}\right)_{\odot}=+4.79,\left(T_{e}\right)_{\odot}=5750^{\circ} \mathrm{K}$. The change with temperature in $M_{\mathrm{bol}}$ and in the bolometric correction, B.C., is negligible,

\footnotetext{
1 The zero point of the bolometric-correction scale is subject to some uncertainty. In a private communication, H. L. Johnson has pointed out a range of corrections to his scale from 0.00 to 0.13 mag. The correction used here makes B.C. for the Sun $-0.05 \mathrm{mag}$; Johnson (1965a) uses for the Sun $m_{V}=$ -26.73 and $M_{\mathrm{bol}}=+4.84$. The range of uncertainty of the solar effective temperature makes both of these plausible, although Johnson's definition makes B.C. $=0.00$ for the Sun.
} 
about \pm 0.08 . The range of $\log R$ is also \pm 0.08 , a more serious uncertainty for theoretical discussion.

\section{EARLIER-TYPE M. DWARFS}

The same method can be applied to the measures by Johnson (1965a), who gave colors from $U$ to $K$ for many late $M$ dwarfs, and $L$ for a few. His analysis was based on mean colors of stars grouped by spectral type (his Table 2), and we reanalyzed his group means as we did W359 and VB 10. However, certain inconsistencies and a rather indeterminate temperature suggested the need for further reanalysis. We are interested only in the faintest end of the main sequence, where the spectral types are poorly defined and the

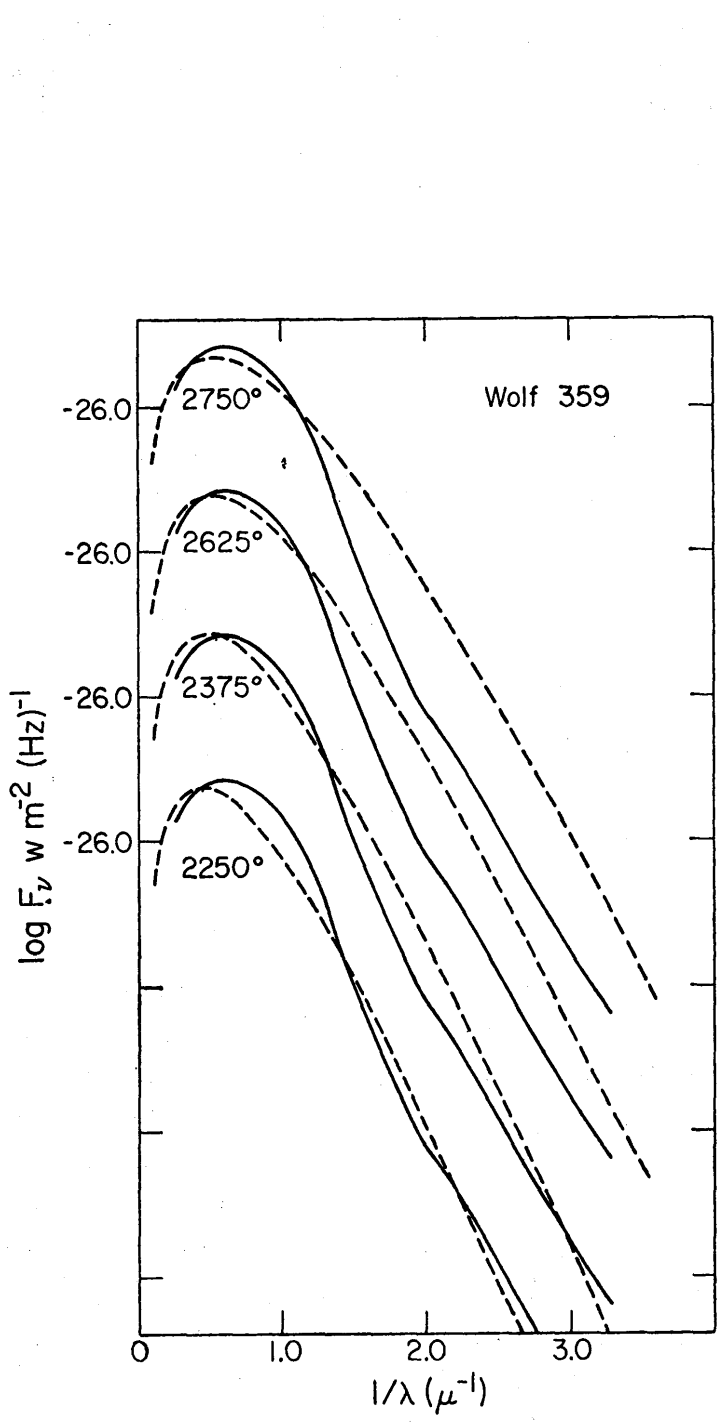

FIG. 2

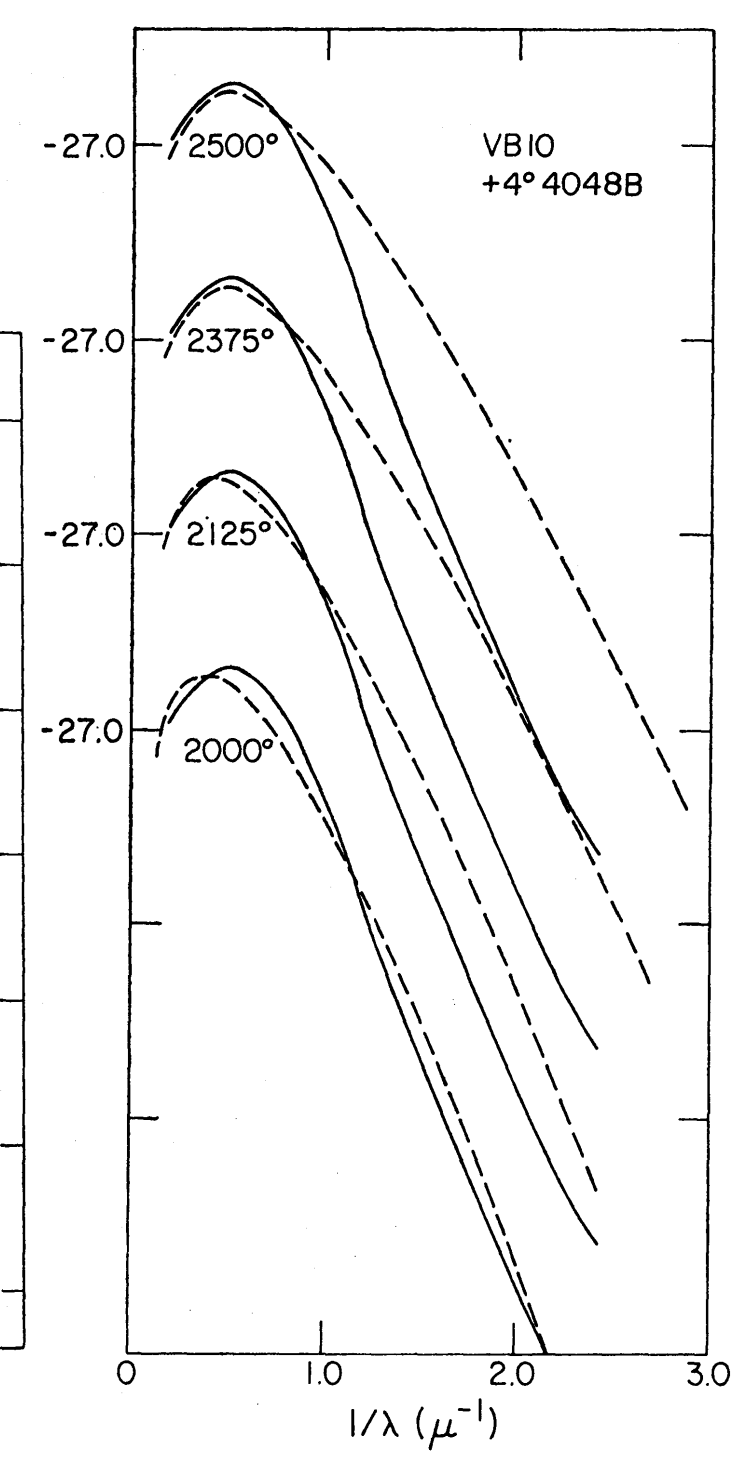

Fig. 3

Fig. 2.-Energy distribution in W359 with four fitted blackbody curves. Vertical scale is shifted to minimize overlap for the different $T: T_{u}=2750^{\circ} \mathrm{K}$ has excessively large depression, $T_{l}$ has no blocking at $\lambda<0.6 \mu$, and a clearly shifted maximum. Best value of $T=2500^{\circ} \mathrm{K}$, between $T_{1}=2625^{\circ} \mathrm{K}$ and $T_{2}=2375^{\circ} \mathrm{K}$.

FIG. 3.-Energy distribution for VB 10, with four fitted blackbody curves. $T_{u}=2500^{\circ}$ clearly requires too much blocking, and $T_{l}$ too little. The best $T=2250^{\circ} \mathrm{K}$. 
slope of the H-R diagram very steep. We regrouped his stars by the $I$-band absolute magnitude $M_{I}$. We used stars of types M3 and later. In Table 4 the new groups are listed, together with their mean colors (see also Fig. 4). The mean spectral types were used only to label the groups; e.g., JM 4.9 has a mean type dM4.9. The parallaxes are large and relatively accurate (averaging 0.2 ; only one is less than 0 ".1). The groups by $M_{I}$ give: JM 4.0, $M_{I}=+7.62$ (five stars); JM 4.9, $M_{I}=+8.62$ (six stars); JM 5.6, $M_{I}=$ +9.92 (seven stars). However, the last group proved unsatisfactory, with a large range of $M_{I}$ and color. A final, more cohesive group, omitting the faint objects Y2582B and Y2890AB and Barnard's star Y4098, was used and called JM 5.3 with $M_{I}=+9.46$ (four stars). No $T$ or $M_{\text {bol }}$ will be given for JM 5.6. The improvement in the shape of the spectral-energy curves is marked. (Barnard's star does not differ markedly from the final mean.) The differences in color from group to group are somewhat erratic, and the Johnson curves do not fit as well as do the individual stars newly observed by us. Perhaps the use of mean colors for stars of mixed population types may be dangerous, since the

TABLE 3

PROPERTIES OF FIT: RADII AND LUMINOSITIES

\begin{tabular}{|c|c|c|c|c|}
\hline$T\left({ }^{\circ} \mathbf{K}\right)$ & $-\log R / R_{\odot}$ & $-\log L / L_{\odot}$ & $M_{\text {bol }}$ & Remarks \\
\hline & \multicolumn{4}{|c|}{ Wolf 359} \\
\hline$T_{l}=2250 \ldots \ldots$ & 0.68 & 2.99 & 12.29 & \multirow{2}{*}{$\begin{array}{l}\text { Excess at } I \text { too large } \\
\text { No blocking } \lambda<0.5 \mu \\
\text { Best } \\
\phi \text { too large at } 0.6 \mu\end{array}$} \\
\hline \multirow[t]{2}{*}{$\begin{array}{l}T_{2}=2375 \ldots \ldots \\
T=2500 \ldots \ldots \\
T_{1}=2625 \ldots \ldots \\
T_{u}=2750 \ldots \ldots\end{array}$} & $\begin{array}{l}0.68 \\
0.73 \\
0.77 \\
0.82\end{array}$ & $\begin{array}{l}2.90 \\
2.90 \\
2.90 \\
2.92\end{array}$ & $\begin{array}{l}12.06 \\
12.04 \\
12.04 \\
12.09\end{array}$ & \\
\hline & \multicolumn{4}{|c|}{ VB 10} \\
\hline $\begin{array}{l}T_{l}=2000 \ldots \ldots \\
T_{2}=2125 \ldots \ldots \\
T=2250 \ldots \ldots \\
T_{1}=2375 \ldots \ldots \ldots \\
T_{u}=2500 \ldots \ldots \ldots\end{array}$ & $\begin{array}{l}0.77 \\
0.81 \\
0.85 \\
0.89 \\
0.93\end{array}$ & $\begin{array}{l}3.37 \\
3.35 \\
3.33 \\
3.32 \\
3.31\end{array}$ & $\begin{array}{l}13.20 \\
13.16 \\
13.12 \\
13.09 \\
13.09\end{array}$ & $\begin{array}{l}\text { No blocking } \lambda<0.8 \mu \\
\text { Best } \\
\phi \text { too large at } 0.7 \mu\end{array}$ \\
\hline
\end{tabular}

$\mathrm{CO}, \mathrm{H}_{2} \mathrm{O}$, and $\mathrm{TiO}$ bands will behave differently as elemental abundances vary. From this point of view it is interesting to find, with large uncertainties, that Barnard's star has $\log L / L_{\odot}=-2.38$ at $\log T_{e}=3.520$, below the JM 5.3 point, i.e., hotter and fainter than the main sequence. The bolometric correction involves the visual luminosity, which according to our assumptions may be strongly depressed. This agrees with the observed great strength of the TiO bands. We give the bolometric correction in Table 5. Note its extremely steep dependence on spectral type. Nevertheless, note also that the change in bolometric correction from type M4 to whatever type VB 10 should be called (M8?) is larger than from the Sun, G2, to M4. The same is true of $M_{V}, M_{\text {bol }}, \log L$, and most color indices. The range of all parameters within the late $M$ dwarfs is very large indeed, and spectral type as now determinable is an insufficiently accurate parameter.

Figure 5 shows the $(L, T)$-diagram for these groups, for W359 and VB 10, for Barnard's star Y4098, and for Kruger 60A and B, taken from Limber (1958a). The data for $\mathrm{Kr} 60$ used by Limber were of complex origin - the six-color system of Stebbins and Whitford, the $(R, I)$-system of Kron, and the old radiometric magnitudes of Pettit and Nicholson. In addition, he used a different solar magnitude. Without attempting to trace the origin and effect of the various differences, we can conclude from Figure 5 that 
TABLE 4

REDISCUSSION, JOHNSON COLORS AND FLUXES

A. COLORS

\begin{tabular}{lllllllll}
\hline \hline & \multicolumn{7}{c}{ MEAN Colors } \\
\cline { 2 - 9 } Group* & $U-V$ & $B-V$ & $V-R$ & $V-I$ & $V-J$ & $V-K$ & $V-L$ & $M_{I}$ \\
\hline JM 4.0... & 2.63 & 1.53 & 1.60 & 2.98 & 3.63 & 4.44 & $4.97:$ & 7.62 \\
JM 4.9. & 2.68 & 1.60 & 1.82 & 3.44 & 4.15 & 4.17 & 5.40 & 8.62 \\
JM 5.6. & $2.99:$ & 1.74 & 1.98 & 3.74 & 4.67 & 5.51 & 5.59 & 9.92 \\
JM 5.3... & $2.98:$ & $1.74:$ & 1.83 & 3.46 & 4.31 & 5.14 & $5.82:$ & 9.46
\end{tabular}

B. FLUXES

\begin{tabular}{|c|c|c|c|c|c|c|c|c|}
\hline \multirow[b]{2}{*}{ Color } & \multicolumn{4}{|c|}{$\left\langle M_{\lambda}\right\rangle$} & \multicolumn{4}{|c|}{$\left\langle-\log F_{\nu}\right\rangle$} \\
\hline & JM 4.0 & JM 4.9 & JM 5.6 & JM 5.3 & JM 4.0 & JM 4.9 & JM 5.6 & JM 5.3 \\
\hline 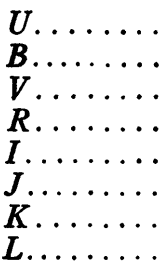 & $\begin{array}{r}13.23 \\
12.13 \\
10.60 \\
9.00 \\
7.62 \\
6.97 \\
6.16 \\
5.63:\end{array}$ & $\begin{array}{c}14.84 \\
13.66 \\
12.06 \\
10.24 \\
8.62 \\
7.91 \\
6.89 \\
6.66:\end{array}$ & $\begin{array}{r}16.65 \\
15.40 \\
13.66 \\
11.68 \\
9.92 \\
8.99 \\
8.15 \\
8.07:\end{array}$ & $\begin{array}{c}\text { 15.90: } \\
14.66: \\
12.92 \\
11.09 \\
9.46 \\
8.61 \\
7.78 \\
7.10:\end{array}$ & $\begin{array}{l}28.02 \\
27.20 \\
26.66 \\
26.14 \\
25.70 \\
25.54 \\
25.66 \\
25.76: \dagger\end{array}$ & $\begin{array}{l}28.67 \\
27.81 \\
27.24 \\
26.64 \\
26.10 \dagger \\
25.91 \dagger \\
25.96 \\
26.17\end{array}$ & $\begin{array}{l}29.39: \dagger \\
28.51 \\
27.88 \\
27.21 \\
26.62 \dagger \\
26.35 \dagger \\
26.46 \\
26.74 \dagger\end{array}$ & $\begin{array}{l}29.12: \\
28.23: \\
27.61 \\
27.00 \\
26.45 \\
26.24 \\
26.35 \\
26.40: \ddagger\end{array}$ \\
\hline
\end{tabular}

* JM 4.0; Y1609, 2267, 2420, 3845AB, 3880.

JM 4.9; Y1668, 1755, 2524, 3296, 3458, 3746.

JM 5. 6; Y49B, 520B, 1305, 2582B, 2730, 2890AB, 4098.

JM 5. 3; Y49B, 520B, 1305, 2730.

$\dagger$ Poor internal agreement within the group.

$\ddagger$ One star only.

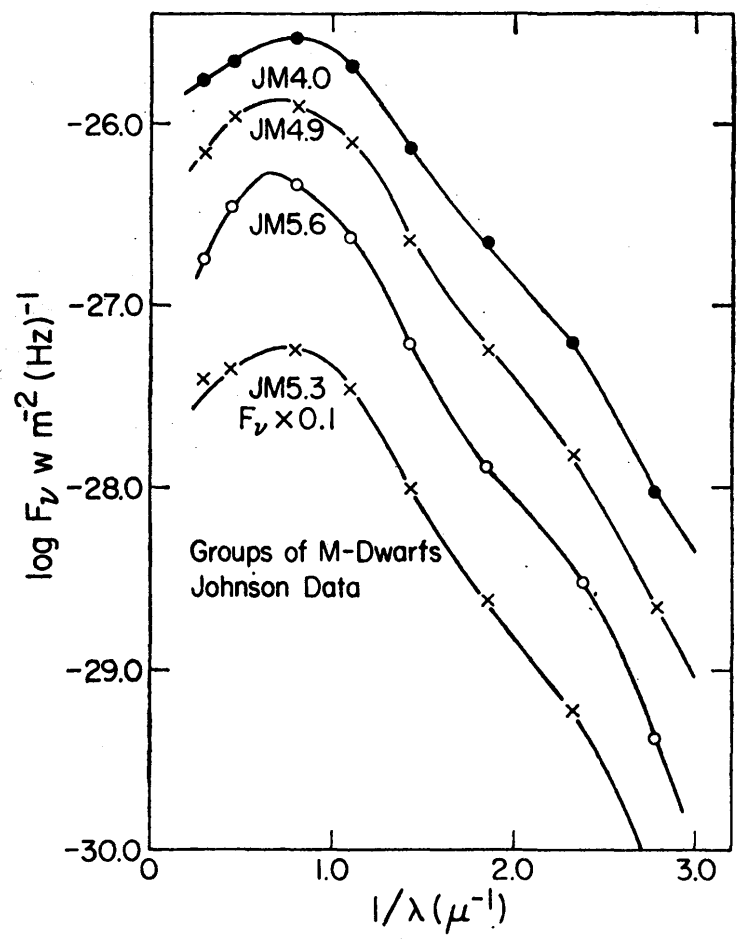

Fig. 4.-Computed fluxes for groups of late-type dwarfs measured by Johnson. The mean types label the groups; JM 5.3 is plotted a factor of 10 lower to reduce interference. 
Limber's results were fundamentally satisfactory. ${ }^{2}$ Since the masses of $\mathrm{Kr} 60 \mathrm{~A}$ and $\mathrm{B}$ are known, this outcome is pleasant even if his model does not reflect the change in stellar energy-generation rates. Limber uses Ross 614B, without any colorimetric data for this faint, nearly unresolvable companion. It is difficult to see what can, in fact, be said about its $L, T$, or $R$. Since its mass is so small $\left(\approx 0.08 \mathfrak{M}_{\odot}\right.$ ), it is an important system for study, but is necessarily omitted here.

A nearly linear relation exists in Figure 5. A straight line forced to fit the Sun and W359 fits the other stars closely. The decrease in $L$ with $T$ is determined as

$$
\log L / L_{\odot}=8.01 \log T-30.12 \text {. }
$$

From the definition of effective temperature and assuming that $T_{e}=T$ (our best value), we find

$$
\log R / R_{\odot}=2.00 \log T-7.54 \text {. }
$$

TABLE 5

RESUME OF TEMPERATURES, LUMINOSITIES, RADII, AND COLORS

\begin{tabular}{lccccccccr}
\hline \hline $\begin{array}{c}\text { Group or } \\
\text { Star }\end{array}$ & $\log T$ & $M V$ & $M_{\text {bol }}$ & B.C. & $\log L / L_{\odot}$ & $\log R / R_{\odot}$ & $V-I$ & $V-L$ & $B-L$ \\
\hline JM 4.0. & 3.544 & 10.60 & 8.60 & -2.00 & -1.56 & -0.35 & 2.98 & $4.97:$ & 6.5 \\
JM 4.9. & 3.512 & 12.06 & 9.67 & -2.39 & -1.95 & -0.48 & 3.44 & $5.40:$ & 7.0 \\
JM 5.3. & 3.504 & 12.92 & 10.34 & -2.58 & -2.22 & -0.60 & 3.46 & $5.82:$ & 7.6 \\
W359... & 3.398 & 16.62 & 12.04 & -4.58 & -2.90 & -0.73 & 5.2 & 7.8 & 9.8 \\
VB 10... & 3.352 & 18.92 & 13.12 & -5.80 & -3.33 & -0.85 & 5.4 & 9.6 & 11.9 \\
\hline
\end{tabular}

Other lines can be forced through $T_{l}$ and $T_{u}$ and given different results, i.e.,

$$
\begin{aligned}
& \log L / L_{\odot}=7.34 \log T_{l}-27.60, \\
& \log R / R_{\odot}=1.67 \log T_{l}-6.28 ; \\
& \log L / L_{\odot}=9.19 \log T_{u}-34.55, \\
& \log R / R_{\odot}=2.59 \log T_{u}-9.74 .
\end{aligned}
$$

Since we can probably take $\log L / L_{\odot}=3.5 \log \mathfrak{M} / \mathfrak{M A}_{\odot}$, the equations (above) give a mean density for VB 10 as $100 \mathrm{~g} \mathrm{~cm}^{-3}$ for $T_{u}, 60 \mathrm{~g} \mathrm{~cm}^{-3}$ for $T$, and $35 \mathrm{~g} \mathrm{~cm}^{-3}$ for $T_{l}$. The latter seems too low, and the radius from equation (7b) seems too large.

In Figure 6 we collect various properties of the stars as a function of $\log T$. In order to complete the linkage to the Sun, we use Johnson's (1965a) temperature calibration for types K5-M1, with a correction of $-0.05 \mathrm{mag}$ in $\left(M_{\mathrm{bol}}\right)_{\odot}$. The results smoothly join the new calibration of JM 4.0 to JM 5.3 and the individual stars W359 and VB 10 . The visual magnitude, $M_{V}$, has a large run with changing slope. The $M_{\text {bol }}$ is nearly linearly dependent on $T$ except for a possible step in the early $M$ types, perhaps de-

${ }^{2}$ Since this paper was completed, measurements of $\mathrm{Kr} 60 \mathrm{~A}$, B were made from 1.25 to $3.4 \mu$. The blackbody curve fitting was made in the same way, and gave a best fit at $3125^{\circ} \mathrm{K}, \log R / R_{\odot}=-0.36$. Since component B is claimed to be $1.5 \mathrm{mag}$ fainter than A visually, if we assume the same color, the correct radius of $\mathrm{A}$ is $\log R / R_{\odot}=-0.41, \log L / L_{\odot}=-1.88, M_{\mathrm{bol}}=+9.5, M_{V}=+11.8$. The deviations from our Figures 5 and 6 suggest that $\mathrm{A}$ is, in fact, hotter than we derive. Recomputation of $L$ and $T_{e}$ for a star with the mass of $\mathrm{Kr} 60 \mathrm{~A}$ would be interesting, and further observations are planned. We are grateful to Harry Hyland and Harvey Butcher for communicating their unpublished observational data. 
pendent on effects of convection. ${ }^{3}$ The $B-L$ and $V-L$ colors are nearly linear. Note that $V-I$ and the negative of the bolometric correction are almost equal, on the average. A flattening in $V-I$ at $\log T<3.4$ is seen as a steepening in $B-L$ and $V-L$, presumably effects of bands. The frequent use of $V-I$ as a temperature indicator seems justified. Since the TiO bands are strong in $V$, and probably increase monotonically, the behavior is simple. In $B-L$ the behavior is even simpler, and the range larger. $B-L$ could be substituted for a complete set of colors, after improved

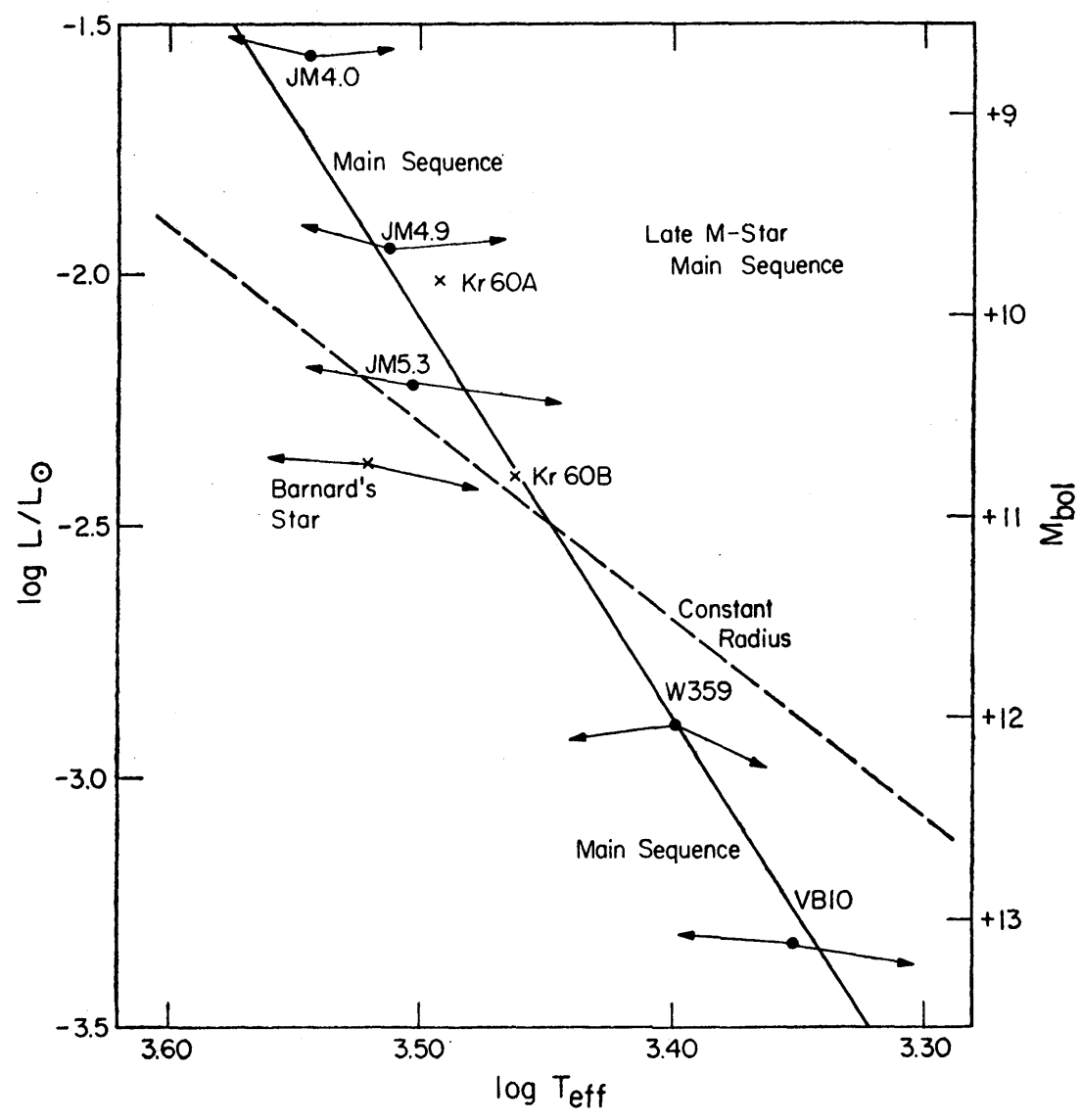

Fig. 5.-Luminosity-effective temperature diagram for the faintest stars. Arrows represent range from $T$ to $T_{u}$ and $T_{l}$. Dashed line, constant-radius locus. Solid line goes through the Sun, at $\log T=$ 3.760. Scale of bolometric magnitudes is based on $\left(M_{\mathrm{bol}}\right)_{\odot}=+4.79$. Barnard's star may be subluminous; Kruger $60 \mathrm{~A}$ and $\mathrm{B}$ are from Limber, without change.

calibration of $T$ for a few other stars cooler than M5. In close binaries with appreciable difference in $T$, the $B-L$ might permit separation of the two components, for example, in Ross 614. We find a mean relation

$$
\log T=3.80-0.04(B-L) .
$$

${ }^{3}$ A recomputation of the lower end of the main sequence has just appeared $(H$. Copeland, J. O. Jensen, and $\mathrm{H}$. E. Jorgensen, $A$ str. and $A$ p., 5, 12, 1970). In this, the effect of $\mathrm{H}_{2}$ molecules on the envelope structure is found to produce just the steepening of the luminosity-temperature curve below $4500^{\circ} \mathrm{K}$ shown in Figure 6. Further, these authors noted an apparent discrepancy between their models and the luminosities observed for $M$ dwarfs of spectral type later than K7. Our higher temperatures reduce this discrepancy, so that their predicted luminosities agree with our observed values at $T=4000^{\circ}$ and $3400^{\circ} \mathrm{K}$. 
But the two stars VB 10 and W359, differing by only $250^{\circ}$, differ by more than 2 mag in $B-L$, much larger than equation (9) predicts.

\section{DISCUSSION}

Clearly, $1-\mu$ to $5-\mu$ measures of faint dwarfs are badly needed and will have important theoretical implications. For example, in spite of a general agreement with Limber's results for $\mathrm{Kr} 60 \mathrm{~A}$ and $\mathrm{B}$, for other stars of known masses no direct check exists since no new infrared colors are available. Barnard's star, with a tangential velocity of $90 \mathrm{~km}$ $\mathrm{sec}^{-1}$ and a radial velocity of $-105 \mathrm{~km} \mathrm{sec}^{-1}$, is a genuine halo star; it may appear too hot because of smaller band and line blocking, or it may be genuinely subluminous (see Fig. 5).

The main result of the present measurement of the two faintest known stars, however, is that we can define the faint end of the main sequence, on the assumption that their $\mathrm{dMe}$ characteristic is irrelevant. Both stars have rather high space motion, about 50

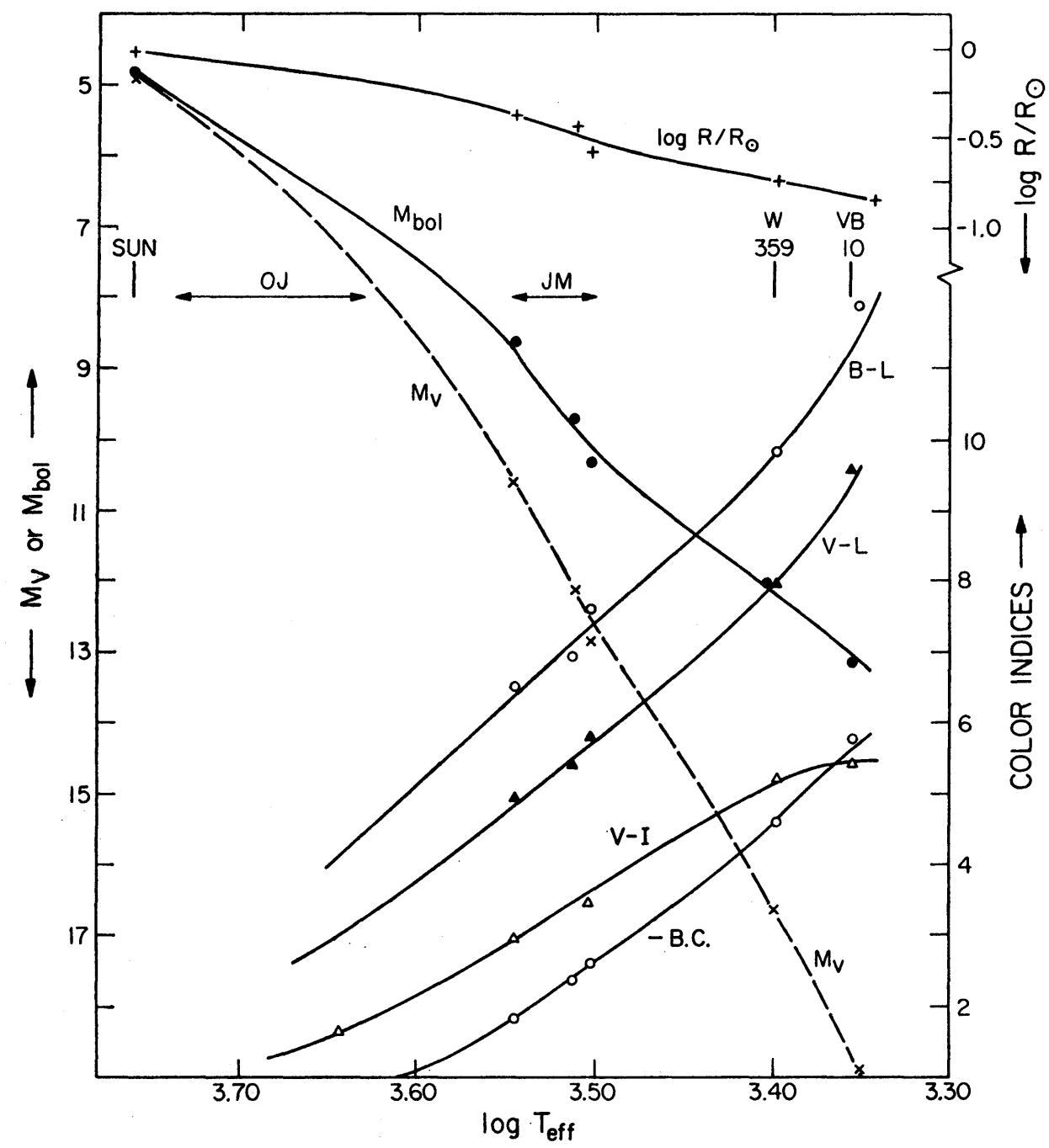

Fig. 6.-Properties of the main sequence fainter than the Sun, as a function of $\log T$. Horizontal arrows mark range where the older Johnson values (OJ) or the present recalibration (JM) were used; vertical bars, W359 and VB 10 . Left-hand scale is for $M_{V}$ or $M_{\text {bol }}$; right-hand scale, for color indices (B.C. plotted with opposite sign). Top right scale for $\log R / R_{\odot}$. Plus signs, radii; dots, $M_{\text {bol }}$; crosses, $M v$; open circles, B.C.; open triangles, $V-I$; filled triangles, $V-L$; open circles, $B-L$. 
$\mathrm{km} \mathrm{sec}^{-1}$, but so do a number of Johnson's $\mathrm{M}$ dwarfs. The flare characteristics were not detectable as variability in the infrared photometry; and there is no "ultraviolet continuum," as is seen during flares. The two faint stars are cool, but $T \geq 2250^{\circ} \mathrm{K}$ is probable and errors are $\pm 250^{\circ} \mathrm{K}$. The bolometric luminosity is well determined and the bolometric correction enormous, but accurate, $\pm 0.1 \mathrm{mag}$. The bolometric correction is larger than older theoretical estimates would give, since they neglect the depression caused in $M_{V}$ by $\mathrm{TiO}$ bands and the atomic lines. The Johnson colors for M4 and M5 stars provide another calibration point and, with the Sun, determine from colors a reasonably good luminosity-effective temperature diagram.

The mass-luminosity relation, however, still requires recalibration. Harris, Strand, and Worley (1963) give a change in slope of the $\mathfrak{M}-L$ relation at $M_{\text {bol }}=+7.5$, from $L \propto \mathfrak{M}^{4}{ }^{0}$ to $L \propto \mathfrak{M}^{2.8}$. However, this change depends largely on objects with mass less than $0.25 \mathfrak{M}_{\odot}$, e.g., $\mathrm{Kr} 60 \mathrm{~A}$ and $\mathrm{B}$ and Ross 614B. Since we must discard the latter and should eventually revise $\mathrm{Kr} 60 \mathrm{~A}$ and $\mathrm{B}$ slightly, the slope is, in fact, poorly determined for low-mass stars. W359 and VB 10 , near $10^{-3.5} L_{\odot}$, probably have $\mathfrak{M} \leq 0.1 \mathfrak{M}_{\odot}$. In spite of their photographic and visual faintness, they do not approach the masses of the invisible companions of astrometric binaries. A major result of this study is that the faintest stars observed are not, in fact, very faint.

We doubt that this is connected with the dMe characteristics, which here are not a property of youth only, since the space velocities are those of old disk stars. The contraction time to the main sequence, in view of convective early phases, is certainly shorter than the age of the Galaxy. The total photon luminosity in the emission lines might normally be 0.1 of the star's luminosity in the $B$-band, or $10^{-4}$ of the total luminosity of the star (see Table 1), i.e., $2 \times 10^{26} \mathrm{ergs} \mathrm{sec}^{-1}$, or about $10^{5} \mathrm{ergs} \mathrm{cm}^{-2} \mathrm{sec}^{-1}$. The mean level of nonthermal solar activity is estimated by Biermann and Lüst (1960) to be about $10^{5}-10^{6} \mathrm{ergs} \mathrm{cm}^{-2} \mathrm{sec}^{-1}$, except in major flares when it reaches $10^{10} \mathrm{ergs}$ $\mathrm{cm}^{-2} \mathrm{sec}^{-1}$. This is a solar total nonthermal luminosity, from photons and particles, of from $5 \times 10^{27}$ to $5 \times 10^{32} \mathrm{ergs} \mathrm{sec}^{-1}$, i.e., very much larger than the flux in the Balmer and $\mathrm{Ca}$ II lines observed in dMe stars. In dMe flares, with a 2.5-mag brightening, and a larger fraction of the energy in Paschen and Balmer continua, the dMe star might approach $10^{28} \mathrm{ergs} \mathrm{sec}^{-1}$. All these numbers, however, do not suggest that a dMe star can be maintained permanently far above the main sequence. In fact, the acoustic noise generation varies as $\rho\left\langle v^{2}\right\rangle^{3 / 2}\left(v / v_{8}\right)^{5}$, where $v$ is the mean turbulent velocity, $v_{8}$ the velocity of sound, and $\rho$ the density. Since the atmospheric densities of dwarf stars are all similar, the important factor is the Mach number. If dMe chromospheres had higher velocities (or higher temperatures) than the solar chromosphere, we might have a greater input. But the spectra are very similar, even during a flare (Greenstein and Arp 1969), with $\mathrm{He}$ I just excited and hydrogen strengthened with respect to $\mathrm{Ca}$ II. It is possible that the far-ultraviolet emission of the dMe corona is large; it would be an interesting orbitingsatellite experiment to search for the nearby dMe stars.

As for the properties of the faintest known stars, their discovery among faint stars of large proper motion will be very difficult because of the rapid drop in $M_{V}$ shown in Figure 6. Luyten (1967) lists the results of a 48-inch Schmidt survey of stars of eighteenth to twentieth magnitude with unusually large motions. Using the reduced proper motion, $H$, he concludes that the group of 204 stars has a mean $M_{\mathrm{pg}}=+17.7$. He has studied in detail twenty-eight new and old stars of large motion with accordant results, i.e., a mean $M_{\mathrm{pg}}=+17.1$ to +17.8 . If known white dwarfs are omitted, the nine stars of known parallax have $\left\langle M_{\mathbf{p g}}\right\rangle=18.1$ (the faintest of these is VB 10). Using

$$
H=M+5+5 \log \mu,
$$

he finds that for this group $\left\langle H_{\mathrm{pg}}\right\rangle=23.6$, i.e., $H-M=5.5$. If the transverse motion $V_{T}$ is independent of luminosity, and if small statistical corrections can be neglected, we derive $\left\langle M_{\mathrm{pg}}\right\rangle=+19.7$ for his stars, with the largest $\left\langle H_{\mathrm{pg}}\right\rangle=+25.2$. On Luyten's scale 
of magnitudes, $\mathrm{VB} 10$ has $M_{\mathrm{pg}}=+20.6$ and $H_{\mathrm{pg}}=+25.2$. Thus VB 10 is like the faintest of his stars, and it will be difficult to find fainter ones. His Palomar Schmidt survey goes very faint, and his stars have $m_{\mathrm{pg}} \approx 21.5$ and $\mu \approx 0$ ". 7 year $^{-1}$. The very faint stars with $\left\langle H_{\mathrm{pg}}\right\rangle \approx+25.2$ are rare-there are only four in Luyten's (1967) Table III. The tangential velocity $V_{T}$ is

$$
5 \log V_{T}=3.38+\left(H_{\mathrm{pg}}-M_{\mathrm{pg}}\right) .
$$

The $H_{\mathrm{pg}}-M_{\mathrm{pg}}=4.6$ for VB 10, i.e., $V_{T}=40 \mathrm{~km} \mathrm{sec}^{-1}$. As a minimal estimate, for stars well scattered over the sky, $V_{T}$ should be larger than the solar motion, $20 \mathrm{~km} \mathrm{sec}^{-1}$, so that $\boldsymbol{H}_{\mathrm{pg}}-\boldsymbol{M}_{\mathrm{pg}}>3.12$. Therefore, the Luyten stars are brighter than $\boldsymbol{M}_{\mathrm{pg}}=$ +22.1. Given the steady run of colors in Figure 6, we may estimate for the faintest stars, $M_{V} \leq+19.8, \log T=3.34$, bolometric correction $=-6.0, M_{\text {bol }} \leq+13.8$, or $\log L / L_{\odot} \geq-3.60$. We are assuming that the run of $T$ in Figure 6 can be slightly extrapolated, and that the infrared colors of Luyten's new, fainter stars resemble those of W359 and VB 10. If $V_{T}$ is larger (say, Population II), the stars are brighter in $M_{\mathrm{pg}}$. The unknown changes in color caused by a weakening of bands and lines in Population II would probably diminish the bolometric correction. But for $V_{T}=200 \mathrm{~km} \mathrm{sec}^{-1}$ (5 times that of old disk stars), the increase in $H-M$ corresponding to a decrease of $M_{\mathrm{pg}}$ by 3.5 should be only partially compensated for by a reduction in the bolometric correction. It seems very improbable that the reduction in bolometric correction would exceed 3.5 mag, so that the Population II stars probably will not, in fact, alter our conclusions as to the rarity of stars below $\log L / L_{\odot}=-3.60$.

If the mean mass-luminosity relation is near $L=\mathfrak{M}^{3.5}$, these stars are more massive than $\log \mathfrak{M}=-1.03$, or $0.093 \mathfrak{M}_{\odot}$, very close to the usual theoretical limit for stars on the main sequence. Less massive stars could be contracting to a degenerate, hydrogenrich configuration with no energy generation. If the Hayashi contraction time is not too long, these stars will be rare, and not necessarily very faint. Much less massive objects would be solids or planets, as are the suspected low-mass astrometric companions.

At the very faint end of the main sequence, certain new difficulties appear. The radius of a completely degenerate, zero-temperature, hydrogen sphere is, from the properties of an $n=\frac{3}{2}$ polytrope,

$$
\log R / R_{\odot}=-\frac{1}{3} \log \mathfrak{M} / \mathfrak{M}_{\odot}-1.40 .
$$

Note that, for $0.1 \mathfrak{M}_{\odot}, \log R / R_{\odot}=-1.07$, i.e., only slightly less than the -0.85 value for VB 10. At equal effective temperatures there is only $1.2 \mathrm{mag}$ difference in bolometric magnitude between main-sequence stars and degenerate configurations. We have neglected the departure from zero temperature for the degenerate star and the finite radius of its nondegenerate envelope, both of which make it larger, and less distinguishable from the main-sequence stars. Detailed models of low-mass stars with and without energy generation may suggest distinguishing characteristics. The degenerate star has small heat content; for a central temperature of $10^{6}{ }^{\circ} \mathrm{K}$ the thermal energy for a gas is only $10^{46} \mathrm{ergs}$, and the cooling time scale about $2 \times 10^{8}$ years. Low-mass stars, without energy generation, will therefore have short lifetimes, and be less common at a given luminosity, than their initial formation rate would require. The presence of a few lowmass stars like L726-8A and B (LTT 892, 893) (see Worley 1969) in the process of contraction might be expected. But with the sum of the masses for that system of 0.08 $\mathfrak{M}_{\odot}$, we face a difficult problem. The proper motion is 3 ".36 year-1, the parallax 0 ".079; the type is dM6e, and the fainter star LTT 893 is a flare star with $m_{\mathrm{pg}}=14.7$, i.e., $M_{\mathrm{pg}}=+14.2$. The tangential velocity is $200 \mathrm{~km} \mathrm{sec}^{-1}$, i.e., that of a Population II star in the halo. If the bolometric correction derived in this paper applies for late-type halo stars, LTT 893 is very overluminous for its mass, i.e., $M_{\text {bol }}=+9.0$. Further, it cannot have been recently formed, given its high velocity. The potential energy of a degenerate, 
low-mass star is $-6 G \mathfrak{M}^{2} / 7 R$; for $\mathfrak{M}=0.04 \mathfrak{M}_{\odot}, R=0.1 R_{\odot}$ (eq. [12]), this is $5 \times 10^{46}$ ergs. Therefore, the total energy release in a Hayashi contraction, even if the star was never brighter than $M_{\text {bol }}=+9$, will suffice for only $2 \times 10^{7}$ years. Thus the existence of relatively bright stars like L726-8A and B, if the mass is correct, which are capable of large flares but which seem to be old, is indeed paradoxical. Since the bolometric correction used here is so large, it is urgently necessary to obtain the infrared colors of the various types of low-luminosity, low-mass stars. Furthermore, since proper-motion surveys to a fixed visual magnitude select stars of relatively high temperatures because of the large bolometric correction, a special survey at $1 \mu$ may be important for a study of the very faint, low-mass stars.

The experimental work was supported by a National Aeronautics and Space Administration grant NGL 05-022-007; the analysis was largely carried out by one of us (J. L. G.) while at the Institute for Advanced Study, and later under AFOSR grant 69-1401.

\section{REFERENCES}

Auman, J. R. 1969, in Low-Luminosity Stars, ed. S. S. Kumar (New York: Gordon \& Breach), p. 483.

Becklin, E. E. 1968, unpublished Ph.D. thesis, California Institute of Technology.

Biermann, L., and Lüst, R. 1960, in Stellar Atmospheres, ed. J. L. Greenstein (Chicago: University of Chicago Press), p. 260.

Greenstein, J. L. 1969, in Low-Luminosity Stars, ed. S. S. Kumar (New York: Gordon \& Breach), p. 281.

Greenstein, J. L., and Arp, H. C. 1969, Ap. J. (Letters), 3, L149.

Harris, D. L., Strand, K. A., and Worley, C. E. 1963, in Basic Astronomical Data, ed. K. Aa. Strand (Chicago: University of Chicago Press), p. 285.

Herbig, G. H. 1956, Pub. A.S.P., 68, 531.

Johnson, H. L. 1964, Bol. Obs. Tonantzintla y Tacubaya, 3, 305. $1965 a, A$. $J ., 141,170$.

1965b, Comm. Lunar and Planet. Lab., 3, 73.

Kandel, R. S. 1969, in Low-Luminosity Stars, ed. S. S. Kumar (New York: Gordon \& Breach), p. 511

Kron, G. E. 1958, Pub. A.S.P., 70, 102.

Limber, D. N. 1958a, Ap.J., 127, 363.

- $1958 b$, ibid., p. 387.

Luyten, W. J. 1967, in Modern Astrophysics, ed. M. Hack (Paris: Gauthier-Villars), p. 149.

Van Biesbroeck, G. 1944, A.J., 51, 61. 1961, ibid., 66, 528 .

Wing, R. F., and Ford, W. K. 1969, preprint.

Worley, C. E. 1969, in Low-Luminosity Stars, ed. S. S. Kumar (New York: Gordon \& Breach), p. 122. 
